PROCEEDINGS OF THE

AMERICAN MATHEMATICAL SOCIETY

Volume 129, Number 9, Pages 2535-2540

S 0002-9939(01)05877-4

Article electronically published on February 22, 2001

\title{
A SIMPLE PROOF OF SOME GENERALIZED PRINCIPAL IDEAL THEOREMS
}

\author{
DAVID EISENBUD, CRAIG HUNEKE, AND BERND ULRICH
}

(Communicated by Wolmer V. Vasconcelos)

\begin{abstract}
Using symmetric algebras we simplify (and slightly strengthen) the Bruns-Eisenbud-Evans "generalized principal ideal theorem" on the height of order ideals of nonminimal generators in a module. We also obtain a simple proof and an extension of a result by Kwieciński, which estimates the height of certain Fitting ideals of modules having an equidimensional symmetric algebra.
\end{abstract}

\section{INTRODUCTION}

The "generalized principal ideal theorem" of Bruns, Eisenbud, and Evans says, roughly, that if $N$ is a finitely generated module over a Noetherian local ring $(R, m)$, and $x$ is an element in $m N$, then the order ideal

$$
N^{*}(x)=\{\varphi(x) \mid \varphi \in \operatorname{Hom}(N, R)\}
$$

has height bounded by the "rank" of $N$. (The usual principal ideal theorem, that the height of a proper ideal generated by $n$ elements is at most $n$, is the case where $N$ is free of rank $n$.) The original form of the result was proved for rings containing a field by Eisenbud-Evans ([3]) and in general by Bruns ([1]). We reduce the statement to the classical principal ideal theorem, applied in the symmetric algebra of a closely related module. In this way we obtain a simple proof of a stronger version.

Our proof also yields a new result on the possible height of the ideal generated by the entries of a row of a matrix whose cokernel $M$ has an equidimensional symmetric algebra. As a consequence we deduce a result of Kwieciński (8] ) which gives a bound for a Fitting ideal $\operatorname{Fitt}_{j}(M)$, if $\sqrt{\operatorname{Fitt}_{j}(M)} \neq \sqrt{\mathrm{Fitt}_{j+1}(M)}$ and the ring is a regular domain (Kwieciński's original proof requires the ring to be a regular affine domain of characteristic 0 ). We also come up with a correction term that allows us to drop the hypothesis on radicals.

For further results in the spirit of this paper, see Johnson ([7]).

One of the drawbacks of the generalized principal ideal theorem stated here is the requirement that $x \in m N$. In a subsequent paper ([4]) we will show that this requirement can often be removed or weakened.

Received by the editors September 21, 1999 and, in revised form, January 14, 2000.

2000 Mathematics Subject Classification. Primary 13C15, 13C40; Secondary 13D10.

Key words and phrases. Height, order ideals, determinantal ideals, symmetric algebras, equidimensionality.

The authors are grateful to the NSF and to MSRI for support.

(C)2001 American Mathematical Society 
We recall that the applications of the generalized principal ideal theorem include two useful statements on the determinantal ideal $I_{t}(\varphi)$ of $t \times t$ minors of an $n \times m$ matrix $\varphi$ that generalize the well-known height formulas due to Macaulay and Eagon-Northcott:

a) If $R$ is local, $I_{t}(\varphi)=0$ and $\varphi^{\prime}$ is the $n \times(m+1)$ matrix derived from $\varphi$ by adding one column with entries in the maximal ideal, then ht $I_{t}\left(\varphi^{\prime}\right) \leq n-t+1$ (Eisenbud-Evans, 3]).

b) If $I_{t+1}(\varphi)=0$, then ht $I_{t}(\varphi) \leq m+n-2 t+1$ (Bruns, [1]).

See the original papers for variations and consequences.

In another paper ([5]) we will exploit symmetric and Rees algebra techniques further to give new bounds for the height of determinantal ideals under additional assumptions such as the ring being regular, or the cokernel of the matrix being torsion free.

For general notions in commutative algebra, see 2]. A good reference for properties of the symmetric algebra is [10].

We started thinking about generalized principal ideal theorems again because of Kwieciński's interesting paper [8], and we would like to thank him for sharing it with us.

\section{Symmetric ALGebras AND ORDER IDEALS}

Lemma 2.1. Let $R$ be a Noetherian ring and let $M$ be a finitely generated $R$ module, with finite free presentation

$$
F_{1} \stackrel{\varphi}{\longrightarrow} F_{0} \longrightarrow M \rightarrow 0 .
$$

Let $\left\{T_{1}, \ldots, T_{n}\right\}$ be a basis of $F_{0}$. Let $b: F_{0} \longrightarrow R$ be any map. If $P \subset R$ is a prime ideal containing image $(b \varphi)$, the "generalized row ideal of $\varphi$ corresponding to $b "$, then

$$
\operatorname{dim}(R / \text { image }(b \varphi))_{P} \geq \operatorname{dim} \operatorname{Sym}(M)_{\left(P,\left\{T_{i}-b\left(T_{i}\right)\right\}\right)}-n .
$$

Proof. The equality

$$
(R / \text { image }(b \varphi))_{P}=\operatorname{Sym}(M)_{\left(P,\left\{T_{i}-b\left(T_{i}\right)\right\}\right)} /\left(\left\{T_{i}-b\left(T_{i}\right)\right\}\right)
$$

shows that the $n$-generator ideal $\left(\left\{T_{i}-b\left(T_{i}\right)\right\}\right)$ is proper. The dimension estimate follows since the ring $\operatorname{Sym}(M)_{\left(P,\left\{T_{i}-b\left(T_{i}\right)\right\}\right)}$ is local (see [2, Corollary 10.9]).

For an $R$-module $M$ and a prime $P \subset R$ we write $\mu_{P}(M)$ for the minimal number of generators of the $R_{P}$-module $M_{P}$. If $(R, m)$ is local, we set $\mu(M)=\mu_{m}(M)$.

Here is the promised strengthening of the generalized principal ideal theorem of Bruns, Eisenbud, and Evans ([1, Theorem 1]):

Theorem 2.2. Let $(R, m)$ be a Noetherian local ring and let $N$ be a finitely generated $R$-module. If $x \in m N$, then $\operatorname{dim} R / N^{*}(x) \geq \operatorname{dim} R / Q-\mu_{Q}(N)$ for every minimal prime $Q$ of $R$; thus for every such $Q$,

$$
\text { ht } N^{*}(x) \leq \operatorname{dim} R-\operatorname{dim} R / Q+\mu_{Q}(N) .
$$

Note that if $R$ is a domain then the right-hand side of the inequality is the rank of $N$. The improvement over the original versions is in the treatment of the rank in the general case. For the proof we need: 
Lemma 2.3. Let $R$ be a Noetherian local ring, let $N$ an $R$-module, and let $\pi$ : $F \longrightarrow N$ be a surjection from a finitely generated free module. If $M=\operatorname{coker}\left(\pi^{*}\right)$, then

$$
\mu(F) \leq \mu(M)+\mu(N) .
$$

Proof of Lemma 2.3. The kernel of $\pi$ contains a free summand $G$ of $F$ with rank $\mu(F)-\mu(N)$. The projection map $F^{*} \longrightarrow G^{*}$ factors through $M$, so $\mu(M) \geq$ $\mu\left(G^{*}\right)=\mu(F)-\mu(N)$.

Proof of Theorem 2.2, Let

$$
F \stackrel{\pi}{\longrightarrow} N \rightarrow 0
$$

be a surjection from a free module $F$ of rank $n=\mu(N)$ onto $N$, and let $M=$ $\operatorname{coker}\left(\pi^{*}\right)$. Let $b \in m F$ be an element mapping onto $x$; we regard $b$ as a map from $F^{*}$ to $R$. Choose a finite free presentation of $M$ of the form

$$
F_{1} \stackrel{\varphi}{\longrightarrow} F^{*} \longrightarrow M \rightarrow 0 .
$$

The order ideal of $x$ is given by $N^{*}(x)=$ image $(b \varphi)$, so we may apply Lemma 2.1 Since all $b\left(T_{i}\right) \in m$ we get

$$
\operatorname{dim} R / N^{*}(x) \geq \operatorname{dim} \operatorname{Sym}(M)_{\left(m,\left\{T_{i}\right\}\right)}-n .
$$

As $\operatorname{Sym}(M)$ is positively graded over a local ring we may use the dimension formula of Huneke and Rossi ([6, Theorem 2.6], [10, Theorem 1.2.1]) to obtain

$\operatorname{dim} \operatorname{Sym}(M)_{\left(m,\left\{T_{i}\right\}\right)}=\operatorname{dim} \operatorname{Sym}(M)=\max \left\{\operatorname{dim} R / Q+\mu_{Q}(M) \mid Q \in \operatorname{Spec}(R)\right\}$.

By Lemma 2.3, $\mu_{Q}(M) \geq n-\mu_{Q}(N)$. Combining these formulas, we conclude that $\operatorname{dim} R / N^{*}(x) \geq \operatorname{dim} R / Q-\mu_{Q}(N)$ for all primes $Q$, and hence in particular for all minimal primes, as required for the first statement. The second statement follows at once.

\section{Heights of DEterminantal ideals}

The formulas for the heights of determinantal ideals that follow from Theorem 2.2 are often sharp. But under an additional hypothesis on $M$, Kwieciński (8 Theorem 1]) gave a remarkable new bound on the heights of the Fitting ideals of $M$. (Recall that, with notation as in Lemma 2.1 the $i^{\text {th }}$ Fitting ideal $\operatorname{Fitt}_{i}(M)$ is $I_{n-i}(\varphi)$.) His proof required $R$ to be a regular affine domain over a field of characteristic 0 , but using Lemma 2.1 we can remove this restriction and prove a stronger result.

We deduce our theorem from a more precise version, giving an estimate for the heights of the generalized row ideals of $\varphi$ :

Theorem 3.1. Let $R$ be a Noetherian universally catenary, locally equidimensional ring whose maximal ideals all have the same height. Let $M$ be a finitely generated $R$-module such that $\operatorname{Sym}(M)$ is equidimensional. If $P$ is a minimal prime ideal of the generalized row ideal image $(b \varphi)$ of Lemma 2.1, then ht $P \leq n+\operatorname{ht} Q-\mu_{Q}(M)$ for any prime $Q \subset P$.

Informally one may state this result as: under the given hypothesis, the row ideals behave as though $M$ had projective dimension 1 ([6, Corollary 2.12 and Theorem 2.6]). For the proof we need the next proposition. 
Proposition 3.2. Let $R$ be a Noetherian universally catenary, locally equidimensional ring whose maximal ideals all have the same height. If $A$ is a graded, equidimensional $R$-algebra, finitely generated by elements of positive degrees, and $P \subset R$ is a prime ideal, then the maximal ideals of $A_{P}$ that contract to $P$ all have height equal to $\operatorname{dim} A_{P}$. Moreover, $A_{P}$ is equidimensional.

Proof. We may write $A=S / J$, where $S$ is a positively graded polynomial ring $R\left[T_{1}, \ldots, T_{n}\right]$ and $J \subset S$ is a homogeneous ideal.

We shall first show that $J$ is height-unmixed. Let $Q$ be a (necessarily homogeneous) minimal prime of $J$. Since $S$ is positively graded and $J$ is homogeneous and $A$ is equidimensional, there exists a homogeneous maximal ideal $\mathcal{M}$ of $S$ such that ht $\mathcal{M} / Q=\operatorname{dim} A$. As $\mathcal{M}$ is homogeneous, it has the form $\mathcal{M}=m S+S_{+}$where $m$ is a maximal ideal of $R$. Hence by our assumptions on $R$ we have ht $\mathcal{M}=\operatorname{dim} R+n$. Since $S_{\mathcal{M}}$ is equidimensional and catenary, ht $Q=\operatorname{ht} \mathcal{M}-\operatorname{ht} \mathcal{M} / Q=\operatorname{dim} R+n-$ $\operatorname{dim} A$.

As $J$ is height-unmixed, $J_{P} \subset S_{P}$ has the same property. Therefore $A_{P}=S_{P} / J_{P}$ is equidimensional, because $S_{P}$ is a catenary, equidimensional positively graded ring over a local ring and $J_{P}$ is a homogeneous ideal.

Next let $\mathcal{M} \subset S$ be maximal among the ideals of $S$ that contract to $P$. Since $S_{\mathcal{M}}$ is catenary and equidimensional of dimension $\operatorname{dim} R_{P}+n$ and $J$ is height-unmixed, we obtain $\operatorname{dim} A_{\mathcal{M}}=\operatorname{dim} S_{\mathcal{M}}-\mathrm{ht} J_{\mathcal{M}}=\operatorname{dim} R_{P}+n-\mathrm{ht} J$. Hence all maximal ideals of $A_{P}$ contracting to $P$ have the same height. On the other hand, taking $\mathcal{M}=P S+S_{+}$, we have $\operatorname{dim} A_{\mathcal{M}}=\operatorname{dim} A_{P}$, because $A_{P}$ is positively graded over the local ring $R_{P}$.

Proof of Theorem 3.1. By Proposition 3.2,

$$
\operatorname{dim} \operatorname{Sym}(M)_{\left(P,\left\{T_{i}-b\left(T_{i}\right)\right\}\right)}=\operatorname{dim} \operatorname{Sym}(M)_{P} .
$$

On the other hand, the Huneke-Rossi dimension formula ([6, Theorem 2.6]) implies that

$$
\operatorname{dim} \operatorname{Sym}(M)_{P} \geq \operatorname{dim}(R / Q)_{P}+\mu_{Q}(M) .
$$

Now we can apply Lemma 2.1.

Here is our generalization of Kwieciński's Theorem:

Theorem 3.3. Let $R$ be a regular domain whose maximal ideals all have the same height. Let $M$ be a finitely generated $R$-module such that $\operatorname{Sym}(M)$ is equidimensional. Let $i$ be a positive integer. If $P$ is a minimal prime ideal of the Fitting ideal $\operatorname{Fitt}_{i-1}(M)$ not containing $\operatorname{Fitt}_{i}(M)$, then

$$
\text { ht } P \leq i(i-\operatorname{rank}(M)) \text {. }
$$

Proof. By Proposition 3.2 the algebra $\operatorname{Sym}(M)_{P}$ is again equidimensional, so we may replace $R$ by $R_{P}$, and thus assume that $i=\mu_{P}(M)$. Taking a free presentation of $M$ as in Lemma 2.1 which is minimal, we must show that the ideal $I_{1}(\varphi)$ generated by the entries of a matrix representing $\varphi$ has height $\leq i(i-\operatorname{rank}(M))$. This ideal is the sum of the $i$ ideals generated by the entries of the rows of a matrix representing $\varphi$. By Theorem 3.1 each of these ideals has height $\leq i-\operatorname{rank}(M)$. By Serre's Theorem ([9. Theorem 3, p. V-18]) the heights of ideals in regular local rings are subadditive, and the desired inequality follows. 
The height estimate of Theorem [3.3 does not extend to the case in which $\sqrt{\text { Fitt }_{i-1}(M)}=\sqrt{\text { Fitt }_{i}(M)}$. For instance, if $M$ is a complete intersection ideal in a regular local ring with $n=\mu(M) \geq 3$ and $i=2$, then $\operatorname{htFitt}_{i-1}(M)=n>$ $2=i(i-\operatorname{rank}(M))$. Our next estimate explains and covers this situation. It refines Theorem 3.3 by adding a correction term for the case when the two Fitting ideals have the same radical.

Theorem 3.4. Let $R$ be a regular domain whose maximal ideals all have the same height. Let $M$ be a finitely generated $R$-module such that $\operatorname{Sym}(M)$ is equidimensional. Let $i \geq \operatorname{rank}(M)$ be an integer. If $P$ is a minimal prime ideal of the Fitting ideal Fitt $_{i-1}(M)$, then

$$
\text { ht } P \leq i(i-\operatorname{rank}(M))+\mu_{P}(M)-i \text {. }
$$

Proof. By Proposition 3.2 we may again replace $R$ by $R_{P}$. For $e=\operatorname{rank}(M), n=$ $\mu(M)$ and $\varphi$ a matrix with $n$ rows presenting $M$, we are going to prove that ht $I_{n-i+1}(\varphi) \leq i(i-e)+n-i$.

We may assume that $I_{n-i+1}(\varphi) \neq 0$. For $n-i \leq j \leq n$ let $\varphi_{j}$ be the matrix consisting of the first $j$ rows of $\varphi$ and define

$$
t=\min \left\{j \mid I_{n-i+1}(\varphi) \subset \sqrt{I_{n-i+1}\left(\varphi_{j}\right)}\right\} .
$$

Obviously $n-i+1 \leq t \leq n$.

By Theorem 3.1 the $t^{\text {th }}$ row ideal $J_{t}$ of $\varphi$ has height at most $n-e$. Hence by 9 . Theorem 3, p. V-18] and the definition of $t$,

$$
\text { ht } I_{n-i+1}(\varphi) \leq n-e+\operatorname{ht} I_{n-i+1}\left(\varphi_{t-1}\right) .
$$

There exists a prime ideal $Q$ with $I_{n-i+1}\left(\varphi_{t-1}\right) \subset Q$ and $I_{n-i+1}\left(\varphi_{t}\right) \not \subset Q$. Since $I_{n-i+1}\left(\varphi_{t}\right) \subset I_{n-i}\left(\varphi_{t-1}\right)$ we automatically have $I_{n-i}\left(\varphi_{t-1}\right) \not \subset Q$. Without changing $I_{n-i+1}\left(\varphi_{t-1}\right)_{Q}$ we may therefore assume that, over the $\operatorname{ring} R_{Q}$,

$$
\varphi_{Q}=\left[\begin{array}{c|c|c}
1_{n-i} & \multicolumn{2}{|c}{0} \\
\hline & \varphi^{\prime} & \varphi^{\prime \prime} \\
\cline { 2 - 3 } 0 & 0 & 1_{s-n+i}
\end{array}\right]
$$

where $n-i+1 \leq s \leq n$ and $\varphi^{\prime}, \varphi^{\prime \prime}$ have $n-s$ rows and entries in the maximal ideal of $R_{Q}$.

The symmetric algebra of $\operatorname{Coker}\left(\varphi^{\prime}\right) \cong M_{Q}$ is equidimensional by Proposition 3.2 and therefore Theorem 3.3 (or Theorem 3.1) yields ht $I_{1}\left(\varphi^{\prime}\right) \leq(n-s)(n-s-e)$. On the other hand, $\mu\left(I_{1}\left(\varphi^{\prime \prime}\right)\right) \leq(n-s)(s-n+i)$. As $I_{n-i+1}\left(\varphi_{t-1}\right)_{Q} \subset I_{1}\left(\varphi^{\prime}\right)+I_{1}\left(\varphi^{\prime \prime}\right)$, inequality $(*)$ implies ht $I_{n-i+1}(\varphi) \leq n-e+(n-s)(n-s-e)+(n-s)(s-n+i) \leq$ $i(i-e)+n-i$.

\section{REFERENCES}

[1] W. Bruns: The Eisenbud-Evans generalized principal ideal theorem and determinantal ideals. Proc. Amer. Math. Soc. 83 (1981), 19-24. MR 82k:13010 
[2] D. Eisenbud: Commutative Algebra with a View Toward Algebraic Geometry. Springer Verlag, 1995.

[3] D. Eisenbud and E. G. Evans: A generalized principal ideal theorem. Nagoya Math. J. 62 (1976), 41-53. MR 53:13195

[4] D. Eisenbud, C. Huneke, and B. Ulrich: Order ideals and a generalized Krull height theorem. Preprint.

[5] D. Eisenbud, C. Huneke, and B. Ulrich: Heights of ideals of minors. In preparation.

[6] C. Huneke and M. Rossi: The dimension and components of symmetric algebras. J. Algebra 98 (1986), 200-210. MR 87d:13010

[7] M. Johnson: Equidimensional symmetric algebras and residual intersections. Preprint.

[8] M. Kwieciński: Bounds for codimensions of Fitting ideals. J. Algebra 194 (1997), 378-382. MR 98m:13018

[9] J.-P. Serre: Algèbre locale, multiplicités. Springer Lect. Notes in Math. 11, 1965. MR 34:1352

[10] W. Vasconcelos: Arithmetic of blowup algebras. London Math. Soc. Lect. Notes 195, Cambridge Univ. Press, 1994. MR 95g:13005

Mathematical Sciences Research Institute, 1000 Centennial Drive, Berkeley, CaliFORNIA 94720

E-mail address: de@msri.org

Department of Mathematics, University of Kansas, Lawrence, Kansas 66045

E-mail address: huneke@math.ukans.edu

Department of Mathematics, Michigan State University, E. Lansing, Michigan 48824

E-mail address: ulrich@math.msu.edu 